\title{
UNIQUENESS OF THE TOPOLOGY IN CERTAIN COMPACT GROUPS
}

\author{
BY \\ T. E. STEWART( $\left.{ }^{1}\right)$
}

We are here interested primarily in the question of the relation of the group structure to the topological structure in a compact group. A natural question that arises here is the question of uniqueness, i.e. is there more than one compact topology on such a group? We give in $\$ 3$ a necessary and suffcient condition that the topology be unique in the case of a connected group. Our considerations proceed from a general structure theorem given in $\$ 1$. In $\$ 4$ we give a structural theorem for compact, connected, abelian groups. The relations between compactness and connectedness arise constantly in the above arguments and this relation is definitely established in $\$ 2$.

I would like to take this opportunity to express my appreciation to Dr. $\mathrm{Ky}$ Fan whose generous assistance made this work possible.

1. In this section we develop a structural theorem for compact, connected groups. We include a complete proof of the necessary lemmas though the arguments are standard.

According to $[6$, p. 282] a compact, connected Lie group $G$ can be expressed as $\left(\prod_{i=1}^{n} X_{i} \times T\right) / P$ where each $X_{i}$ is a compact, connected, simply connected, simple, nonabelian Lie group, $T$ is a toral group and $P$ is a finite, central subgroup of the product. We generalize this result as follows:

THEOREM 1.1. If $X$ is a compact, connected group then $X$ can be expressed as

$$
X=\left(\prod_{\nu \in J} X_{\nu} \times T^{*}\right) / P^{*}
$$

where

1. $J$ is a suitable index set.

2. Each group $X$, is a compact, connected, simply connected, simple nonabelian Lie group.

3. $T^{*}$ is a compact, connected, abelian group.

4. $P^{*}$ is a 0-dimensional invariant subgroup of $\prod_{v \in J} X_{v} \times T^{*}$.

We first develop a few preliminary results necessary to prove the above theorem. If $G$ is a connected compact Lie group written as $\prod_{i=1}^{n} X_{i} \times T / P$ we denote by $\eta$ the canonical map of $\prod_{i=1}^{n} X_{i} \times T$ onto $G$.

Received by the editors January 18,1960 .

(1) This work is taken from part of the author's doctoral dissertation submitted to the University of Notre Dame in partial fulfillment of the requirements for the degree of Doctor of Philosophy. 
Lemma 1. Let $G=\left(\prod_{i=1}^{n} X_{i} \times T\right) / P, G^{\prime}=\left(\prod_{j=1}^{n \prime} X_{j}^{\prime} \times T^{\prime}\right) / P^{\prime}$ be two compact, connected Lie groups and let $\phi: G \rightarrow G^{\prime}$ be a continuous homomorphism of $G$ onto $G^{\prime}$. Then $\eta(T)$ is mapped by $\phi$ into $\eta^{\prime}\left(T^{\prime}\right)$ and $\eta\left(\prod_{i=1}^{n} X_{i}\right)$ is mapped by $\phi$ into $\eta^{\prime}\left(\prod_{j=1}^{n^{\prime}} X_{j}^{\prime}\right)$.

Proof. Let $(S)$ and $\left(\mathcal{S}^{\prime}\right.$ be the Lie algebras of $G$ and $G^{\prime}$ respectively. Then $\phi$ induces a homomorphism $\phi:\left(S \rightarrow\left(S^{\prime}\right.\right.$. Now $\eta(T)$ is evidently the connected component of the center of $G$ and thus corresponds to the center $\mathscr{B}$ of $\mathbb{B}$, which is the maximal ideal satisfying $[x, y]=0$ for $x \in \mathfrak{B}, y \in \mathbb{B}$. Since $\phi$ is onto, $\Phi$ is onto and for any $z \in \mathcal{S}^{\prime}, z=\Phi(y)$, and $\phi(x), x \in \mathfrak{B},[\Phi(x), \phi(y)]$ $=\Phi([x, y])=0$. Consequently $\phi(x)$ belongs to the center, $\mathfrak{B}^{\prime}$, of $\mathfrak{S S}^{\prime}$, which of course corresponds to $\eta^{\prime}\left(T^{\prime}\right)$. If $\mathfrak{A}$ denotes the Lie algebra of $\eta\left(\prod_{i=1}^{n} X_{i}\right)$ then $[\mathfrak{A}, \mathfrak{A}]=\mathfrak{A}$ and in fact $[\mathfrak{S}, \mathfrak{S}]=\mathfrak{A}$. Then if $\mathfrak{U}^{\prime}$ is the Lie algebra of $\eta^{\prime}\left(\prod_{i=1}^{n^{\prime}} X_{i}^{\prime}\right)$, $\mathfrak{P}^{\prime}=\left[\mathfrak{U}^{\prime}, \mathfrak{P}^{\prime}\right]=[\boldsymbol{\phi}(\mathfrak{Y}), \bar{\phi}(\mathfrak{A})]=\Phi[\mathfrak{A}, \mathfrak{A}]=\bar{\phi}(\mathfrak{A})$ and the lemma follows from the canonical correspondence of Lie groups and their Lie algebras.

LEMMA 2. With the hypothesis as in Lemma 1, $\phi$ induces a continuous homomorphism, $\Phi$, of $\prod_{i=1}^{n} X_{i} \times T /(T \cap P)$ into $\prod_{j=1}^{n^{\prime}} X_{j}^{\prime} \times T^{\prime} /\left(T^{\prime} \cap P^{\prime}\right)$ and the map $\tilde{\phi}$ is the product map of two maps $\tilde{\phi}_{1}: \prod_{i=1}^{n} X_{i} \rightarrow \prod_{j=1}^{n^{\prime}} X_{j}^{\prime}, \tilde{\phi}_{2}: T /(T \cap P)$ $\rightarrow T^{\prime} /\left(T^{\prime} \cap P^{\prime}\right)$.

Proof. Set $\phi_{1}=\left(\phi \mid \eta\left(\prod X_{i}\right)\right) \eta$. By Lemma $1, \phi_{1}$ maps $\prod_{i=1}^{n} X_{i}$ onto $\eta^{\prime}\left(\prod_{j=1}^{n^{\prime}} X_{j}^{\prime}\right)$. Since $\prod_{j=1}^{n^{\prime}} X_{j}^{\prime}$ is locally isomorphic to $\eta^{\prime}\left(\prod_{j=1}^{n \prime} X_{j}^{\prime}\right), \phi_{1}$ defines a local homomorphism of $\prod_{i=1}^{n} X_{i}$ into $\prod_{j=1}^{n^{\prime}} X_{j}^{\prime}$. Now $\prod_{i=1}^{n} X_{i}$ is simply connected and there exists a unique extension $\tilde{\phi}_{1}: \prod_{i=1}^{n} X_{i} \rightarrow \prod_{j=1}^{n^{\prime}} X_{j}^{\prime}$. Similarly, we have a local homomorphism $\phi_{2}$ defined by $\phi$ from $T /(T \cap P)$ into $T^{\prime} /\left(T^{\prime} \cap P^{\prime}\right)$. It is easily seen that the natural extension $\tilde{\phi}_{2}$ is well defined on these groups. The product of the two homomorphisms $\tilde{\phi}_{1}$ and $\tilde{\phi}_{2}$ is the desired homomorphism $\tilde{\phi}$.

LEMMA 3. With the situation as above, we have $\phi \eta=\eta^{\prime} \tilde{\phi}$.

Proof. It is evident from the construction of $\tilde{\phi}$ that this is true locally and since the groups are connected this relation holds globally.

Proof of Theorem 1.1. Let $X$ be a given compact, connected group. Let $\left\{X_{\alpha}, \phi_{\alpha}^{\beta}\right\}_{\alpha \in S}$ be an inverse system of compact, connected Lie groups with inverse limit $X$ and $\phi_{\alpha}^{\beta}: X_{\beta} \rightarrow X_{\alpha}$ a continuous homomorphism onto for each pair $\alpha, \beta \in S, \alpha<\beta[3$, p. 99]. For each $\alpha \in S$ we choose a fixed representation

$$
X_{\alpha}=\frac{\prod_{i=1}^{n_{\alpha}} X_{i, \alpha} \times T_{\alpha}}{P_{\alpha}}
$$

Applying Lemmas 1 and 2 we obtain an inverse system $\left\{\tilde{X}_{\alpha}, \tilde{\phi}_{\alpha}^{\beta}\right\}$ of compact, connected Lie groups with $\tilde{X}_{\alpha}=\prod_{i=1}^{n_{\alpha}} X_{i, \alpha} \times T_{\alpha} /\left(T_{\alpha} \cap P_{\alpha}\right)$, and $\tilde{\phi}_{\alpha}^{\beta}$ the homomorphism induced by $\phi_{\alpha}^{\beta}$. Since each $\tilde{X}_{\alpha}$ is compact, the inverse limit 
$\tilde{X}_{\infty}$ is also compact. Furthermore, we have each $\tilde{X}_{\alpha}$ the direct product of an abelian and a non-abelian group and the homomorphisms $\tilde{\phi}_{\alpha}^{\beta}$ are product homomorphisms. It follows that $\tilde{X}_{\infty}$ is the direct product $\tilde{X}_{\infty}=\tilde{X}_{\infty}^{1} \times \tilde{X}_{\infty}^{2}$ where $\tilde{X}_{\infty}^{1}$ is the inverse limit of the sequence $\left\{\prod_{i=1}^{n_{\alpha}} X_{i, \alpha}, \tilde{\phi}_{\alpha}^{\beta} \mid \prod X_{i, \alpha}\right\}_{\alpha \in S}$ and $\tilde{X}_{\infty}^{2}$ is the inverse limit of $\left\{T_{\alpha} /\left(T_{\alpha} \cap P_{\alpha}\right), \tilde{\phi}_{\alpha}^{\beta} \mid T_{\alpha} /\left(T_{\alpha} \cap P_{\alpha}\right)\right\}_{\alpha \in S}$. Clearly, $\tilde{X}_{\infty}^{2}$ is a compact, connected abelian group. In order to simplify the notation, we henceforth denote the group $\prod_{i=1}^{n_{\alpha}} X_{i, \alpha}$ by $Y_{\alpha}$ and $\tilde{\phi}_{\alpha}^{\beta} \mid Y_{\alpha}$ by $\bar{\phi}_{\alpha}^{\beta}$. According to Lemma 3, we have a map $\eta$ of the inverse system $\left\{\tilde{X}_{\alpha}, \tilde{\phi}_{\alpha}^{\beta}\right\}$ into $\left\{X_{\alpha}, \phi_{\alpha}^{\beta}\right\}$ and this map induces a continuous homomorphism $\eta_{\infty}$ of $\tilde{X}_{\infty}$ into $X$. Since the kernel of each $\eta_{\alpha}$ is finite and the kernel of $\eta_{\infty}$ is the inverse limit of the kernels of $\eta_{\alpha}$, the kernel of $\eta_{\infty}$ is 0 -dimensional.

We study now the group $\tilde{X}_{\infty}^{1}$ which is the inverse limit of the system $\left\{Y_{\alpha}, \Phi_{\alpha}^{\beta}\right\}_{\alpha \in S}$. For this purpose consider the kernel $B$ of the homomorphism $\Phi_{\alpha}^{\beta}: Y_{\beta} \rightarrow Y_{\alpha}$. Since $\phi_{\alpha}^{\beta}$ is onto we have $Y_{\alpha}=Y_{\beta} / B$. It follows from the simple connectivity of $Y_{\alpha}$ that $B$ is a connected, compact, invariant subgroup of $Y_{\beta}$. By the unique decomposition of a simply-connected compact Lie group by its connected invariant subgroups we have $B=\prod_{i=1}^{n_{\beta}^{-n_{\alpha}}} X_{k_{i}, \beta}$. Then $\bar{\phi}_{\alpha}^{\beta}$ induces an isomorphism of a factor $\prod_{j=1}^{n_{\alpha}} X_{i_{j}, \beta}$ onto $Y_{\alpha}$. Again by the uniqueness of decomposition factors go to factors and isomorphically since these factors are simple and simply connected.

Let $S^{\prime}$ denote the index set consisting of pairs $(i, \alpha), \alpha \in S, 1 \leqq i \leqq n_{\alpha}$. We define the following equivalence relation $R$ on $S^{\prime}$ :

$$
(i, \alpha) \equiv(j, \beta) \bmod R,
$$

if there exists $\gamma \in S$ and $k, 1 \leqq k \leqq n_{\gamma}$ with $\alpha<\gamma, \beta<\gamma$ and

$$
\begin{aligned}
& \phi_{\alpha}^{\gamma}\left(X_{k, \gamma}\right)=X_{i, \alpha}, \\
& \boldsymbol{\phi}_{\beta}^{\gamma}\left(X_{k, \gamma}\right)=X_{j, \beta} .
\end{aligned}
$$

Using $R$ we define the index set $J$ to be the set of equivalence classes in $S^{\prime}$. With each $\nu \in J$ we associate the simple Lie group $X$, which is isomorphic to each of the groups $X_{i, \alpha},(i, \alpha) \in \nu$. We will now define for each $\nu \in J$ and $\alpha \in S$ a homomorphism $\Phi_{\nu}^{\alpha}: X_{\nu} \rightarrow Y_{\alpha}$ satisfying

$$
\Phi_{\nu}^{\alpha}=\Phi_{\alpha}^{\beta} \Phi_{\nu}^{\beta}, \quad \alpha<\beta .
$$

Let $\alpha_{0}$ be an arbitrary, fixed element of $S$ which has a factor $X_{i, \alpha_{0}}$, $\left(i, \alpha_{0}\right) \in \nu$. Define $\Phi_{\nu}^{\alpha_{0}}: X_{\nu} \rightarrow Y_{\alpha_{0}}$ to be any isomorphism which carries $X$, onto the factor $X_{i, \alpha_{0}}$ of $Y_{\alpha_{0}}$. If $\gamma \in S$ and $\alpha_{0}<\gamma$, define $\Phi_{\nu}^{\gamma}: X_{\nu} \rightarrow X_{\gamma}$ by

$$
\Phi_{\nu}^{\gamma}=\left(\Phi_{\alpha_{0}}^{\gamma}\right)^{-1} \Phi_{\nu}^{\alpha_{0}} \text {. }
$$

The inverse $\left(\phi_{\alpha_{0}}^{\gamma}\right)^{-1}$ is well defined as we are in one of the factors. Now if $\beta \in S$, then we define $\Phi_{\nu}^{\beta}$ in the following fashion. There exists $\gamma \in S$ such that $\alpha_{0}<\gamma, \beta<\gamma$. Then we set 


$$
\Phi_{\nu}^{\beta}=\phi_{\beta}^{\gamma} \Phi_{\nu}^{\gamma}
$$

It is not difficult to show that this definition does not depend on $\gamma$ and that (1.2) is satisfied.

Having defined $\Phi_{\nu}^{\alpha}$ for all pairs $(\nu, \alpha)$ we define the map $\Phi^{\alpha}: \prod_{\nu \in J} X_{\nu} \rightarrow Y_{\alpha}$ to be the product homomorphism of $\Phi_{\nu}^{\alpha}, \nu \in J$. (1.2) allows us to pass to the limit to obtain $\Phi^{\infty}: \prod_{\nu \in J} X_{\nu} \rightarrow X_{\infty}^{1}$. It is easily seen that $\Phi^{\infty}$ is an isomorphism onto.

2. We point out here some relations between compactness and connectedness in a topological group. It will be shown that the connected component of the identity of a compact group will be the same subgroup in any compact, group topology put on that group and can in fact be algebraically characterized.

THEOREM 2.1. Let $G$ be a compact group. Let $G^{n}=\left\{x^{n} \mid x \in G\right\}$ for each positive $n$. Then the connected component $G_{0}$ of the identity in $G$ is precisely $\bigcap_{n=1}^{\infty} G^{n}$.

Proof. It is well known that $G_{0} \subset \cap_{n=1}^{\infty} G^{n}$ if $G$ is a Lie group or if $G$ is abelian. It follows easily from the decomposition Theorem 1.1 that $G_{0}$ $\subset \cap_{n=1}^{\infty} G_{0}^{n} \subset \cap_{n=1}^{\infty} G^{n}$. Let $a \in \cap_{n=1}^{\infty} G^{n}$ and denote by $\mu: G \rightarrow G / G_{0}$ the canonical homomorphism. We will show $\mu(a)$ is the identity of $G / G_{0}$. Since $G / G_{0}$ is 0 -dimensional, there exists arbitrarily small open subgroups $H_{\nu}, \nu \in J,[5$, p. 56] of $G / G_{0}$ such that $\left(G / G_{0}\right) / H_{\nu}$ are finite groups, and consequently there exists $n_{\nu}$ such that for all $\alpha \in G / G_{0}, \alpha^{n_{\nu}} \in H_{\nu}$. Since $a \in \bigcap_{n=1}^{\infty} G^{n}$, there exists $y \in G$ such that $y^{n_{\nu}}=a$. Then $\mu(a)=\mu\left(y^{n_{\nu}}\right)=\mu(y)^{n_{\nu}}$ is in $H_{\nu}$. Consequently $\mu(a) \in \bigcap_{\nu \in J} H_{\nu}=e$, the identity of $G / G_{0}$. Thus $a \in G_{0}$ and $G_{0}=\bigcap_{n=1}^{\infty} G^{n}$.

3 . We propose to study here some of the relations between the group structure and the topology of compact groups. Since we will be considering a group and various topologies on that group, we will use the following conventions. We denote an abstract group by $G, X$, etc. We write $G(\mathbb{E})$ for the topological group with underlying group $G$ and topology $\mathfrak{E}$. $\prod_{v \in J} X_{\nu}\left(\mathfrak{E}_{\nu}\right)$ will mean the product group in the usual product topology. If $\alpha: G \rightarrow X$ is a homomorphism, then $\alpha$ considered as a (not necessarily continuous) function from $G(\mathfrak{S})$ to $X\left(\mathfrak{S}_{1}\right)$ will be denoted by $\alpha\left(\mathfrak{S}, \mathfrak{S}_{1}\right)$. We mention that if there is no possibility of confusion we will lapse into the usual notation. In this section our main interest will be the uniqueness of a compact topology on a group. We will see that uniqueness largely depends on the center.

THEOREM 3.1. Let $G(\mathfrak{S})$ be a compact, connected group with a totally disconnected center. If $\mathfrak{G}_{1}$ is another topology such that $G\left(\mathfrak{G}_{1}\right)$ is a compact topological group, then $\mathfrak{S}_{1}$ coincides with $\mathbb{E}$.

We first show some preliminary results that will lead the way to the proof of the above theorem.

It is easily seen that the hypothesis on the center and the decomposition 
theorem of the preceding section provide a representation of $G(\mathbb{S})$ as:

$$
G(\mathfrak{S})=\prod_{\nu \in J} X_{\nu}\left(\mathfrak{\mho}_{\nu}\right) / P(\mathfrak{E})
$$

where for each $\nu \in J, X_{\nu}\left(\mathbb{E}_{v}\right)$ is a simple, simply connected, compact Lie group and $P(\mathfrak{E})$ is a 0 -dimensional, central subgroup. We now establish:

Lemma 3.2. Let $G(\mathfrak{S})=\prod_{\nu \in J} X_{\nu}\left(\mathfrak{S}_{\nu}\right)$ where $X_{\nu}\left(\mathfrak{G}_{\nu}\right), \nu \in J$, is as above and furthermore has a degenerate center, then Theorem 3.1 is true for $G(\mathbb{E})$.

Proof. Let $\mathfrak{G}_{1}$ also be a topology such that $G\left(\mathfrak{G}_{1}\right)$ is a compact group. Consider the projection $p_{\nu}: G \rightarrow X_{\nu}$. We will first show that the kernel of $p_{\nu}$ is a closed subgroup of $G\left(\mathcal{E}_{1}\right)$. Set $B_{\nu}=\left\{x \in G \mid x_{\mu}=e, \mu \neq \nu\right\}$. For each $y \in B_{\nu}$ define the continuous function $\phi_{y}: G\left(\mathfrak{S}_{1}\right) \rightarrow G\left(\mathfrak{S}_{1}\right)$ by $\phi_{y}(x)=x y x^{-1} y^{-1}$. Since $\phi_{y}$ is continuous, the set $E_{y}=\left\{x \in G \mid \phi_{y}(x)=e\right\}, e$ the identity of $G$, is a closed set. Now consider the closed set $E=\bigcap_{y \in B_{\nu}} E_{y}$. Clearly an element of $G$ is in $E$ if and only if its $\nu$ th component is in the center of $X_{\nu}$. Since we have assumed this center to be degenerate, it follows that the kernel of $p_{\nu}$ is precisely $E$ and hence closed. Then $p_{\nu}$ induces from $\mathfrak{E}_{1}$ a compact topology on $X_{\nu}$. According to $[6, p .782]$, the only invariant subgroups of $X_{\nu}$ are contained in the center which is trivial. It follows that this topology gives $X_{\nu}$ as a simple, compact, connected Lie group. Applying the results of $[8$, p. 784] which state that there is only one such topology on $X_{\nu}$, we have the induced topology on $X_{\nu}$ from $G\left(\mathfrak{E}_{1}\right)$ the same as $\mathfrak{E}_{\nu}$. Define $\mu\left(\mathfrak{S}_{1}, \mathfrak{E}\right): G\left(\mathfrak{S}_{1}\right) \rightarrow G(\mathfrak{E})$ by $\mu(x)=\left\{p_{\nu}(x)\right\}$. Since each coordinate function $p_{\nu}$ is continuous $\mu$ is continuous. As a function $\mu$ is the identity and it follows from the compactness of $G\left(\mathfrak{G}_{1}\right)$ that $\mathfrak{G}_{1}$ coincides with $\mathcal{~}$.

We return now to the general case. Referring to (3.1) we define $P_{\mu}(\mathbb{E})$ to be the image of $P(\mathfrak{S})$ under the projection $\prod_{\nu \in J} X_{\nu}\left(\mathfrak{S}_{\nu}\right) \rightarrow X_{\mu}\left(\mathfrak{S}_{\mu}\right)$. Apparently $P_{\mu}(\mathbb{S}), \mu \in J$, is a finite subgroup of $X_{\mu}$ contained in the center $C_{\mu}$ of $X_{\mu}$. Define $X_{\nu}^{\prime}\left(\mathfrak{E}_{v}\right)=X_{\nu}\left(\mathfrak{\mho}_{\nu}\right) / C_{\nu}(\mathfrak{夭})$. Set $X(\mathfrak{E})=\prod X_{\nu}\left(\mathfrak{S}_{v}\right)$. The underlying group $X$ apparently has as its center $C=\prod_{\nu \in J} C_{\nu}$, and $C$ will be closed in any admissible topology on $X$. Since $P \subset \prod P_{\nu} \subset C$ we have the following commutative diagram

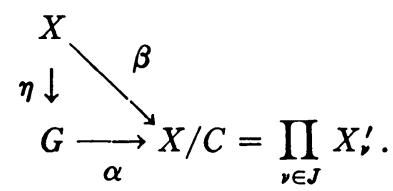

For $a \in X$, let $\bar{a}=a P$ be in the center of $G$. Then for all $d \in X, d a d^{-1} \in P$. Regarding $d \rightarrow d a d^{-1}$ as a continuous function on $X(\mathcal{E})$, the connectedness of $X(\mathfrak{S})$ gives a connected subset of $P$ which then must be $a$ and hence $a \in C$ and consequently the center of $G$ is $\eta(C)$, and is then closed in any admissible topology on $G$. Clearly $\eta(C)$ is the kernel of $\alpha$. 
Now let $\mathfrak{G}_{1}$ be a topology such that $G\left(\mathfrak{夭}_{1}\right)$ is a compact group. Since the kernel of $\alpha$ is a closed subgroup, $\alpha$ induces a compact topology on $X / C$. Now $X / C$ is easily seen to be a group described in the hypothesis of Lemma 3.2. Then this induced topology gives $X / C$ as $\prod_{v \in J} X_{v}^{\prime}\left(\mathbb{E}_{v}\right)$ and in the diagram (3.2) we have $\alpha\left(\mathcal{E}_{1}, \mathfrak{E}\right)$ and $\beta(\mathfrak{E}, \mathfrak{E})$ continuous. We wish to show now that $\eta\left(\mathbb{E}, \mathbb{E}_{1}\right)$ is continuous.

Since the group $\eta(C)$ is the kernel of the continuous map $\alpha\left(\mathbb{E}_{1}, \mathbb{E}\right)$, $\eta(C)\left(\mathfrak{E}_{1}\right)$ is a compact group. It follows from Theorem 2.1 that $\eta(C)\left(\mathfrak{G}_{1}\right)$ is a 0-dimensional, compact group. Then $\eta(C)\left(\mathfrak{E}_{1}\right)$ has arbitrarily small open, invariant subgroups $\left\{H_{\mu}^{1}\right\}_{\mu \in M}$. Consequently we have the continuous homomorphisms $\phi_{\mu}: G\left(\mathfrak{S}_{1}\right) / H_{\mu}^{1}\left(\mathfrak{\mho}_{1}\right) \rightarrow G\left(\mathfrak{\mho}_{1}\right) / \eta(C)\left(\mathfrak{G}_{1}\right)=X / C$ and each homomorphism $\phi_{\mu}$ has a finite kernel. Since $X(\mathfrak{E})$ is simply connected the continuous function $\beta(\mathfrak{E}, \mathfrak{E})$ can be covered by a unique continuous function $\eta_{\mu}: X(\mathfrak{E})$ $\rightarrow G\left(\mathfrak{G}_{1}\right) / H_{\mu}^{1}\left(\mathfrak{G}_{1}\right)$ such that $\eta_{\beta}(e)=e$ (e here the identity of both groups) and $\phi_{\mu} \eta_{\mu}=\beta\left[5\right.$, p. 235]. We show first that $\eta_{\mu}$ is a homomorphism. Indeed, for all $x, y \in X, \quad \phi_{\mu}\left(\eta_{\mu}(x y) \cdot \eta_{\mu}(y)^{-1} \eta_{\mu}(x)^{-1}\right)=\beta(x y) \beta(y)^{-1} \beta(x)^{-1}=e$. Hence $\eta_{\mu}(x y) \eta_{\mu}(y)^{-1} \eta_{\mu}(x)^{-1}$ is in the kernel of $\phi_{\mu}$. Since $f_{\mu}(x, y)=\eta_{\mu}(x y) \eta_{\mu}(y)^{-1} \eta_{\mu}(x)^{-1}$ is clearly continuous on $X(\mathfrak{S}) \times X(\mathfrak{S})$ the image under $f_{\beta}$ is connected and hence reduces to the point $e$. If $\psi_{\mu}^{\mu^{\prime}}: G\left(\mathbb{E}_{1}\right) / H_{\mu}{ }^{\prime}\left(\mathfrak{G}_{1}\right) \rightarrow G\left(\mathfrak{S}_{1}\right) / H_{\mu}^{1}\left(\mathfrak{E}_{1}\right)$ for $H_{\mu^{\prime}}^{1} \subset H_{\mu}^{1}$, it follows from the uniqueness of $\eta_{\mu}$ that $\eta_{\mu}=\psi_{\mu}^{\mu^{\prime}} \eta_{\mu^{\prime}}$. Now $\left\{G\left(\mathbb{E}_{1}\right) / H_{\mu}^{1}\left(\mathbb{E}_{1}\right), \psi_{\mu}^{\mu^{\prime}}\right\}_{\mu \in M}$ forms an inverse system whose inverse limit is easily seen to be $G\left(\mathbb{E}_{1}\right)$. The above relations show that $\left\{\eta_{\mu}\right\}$ provide a map of $X(\mathbb{E})$ into this inverse limit and defines a continuous limit homomorphism $\eta_{\infty}: X(\mathbb{E}) \rightarrow G\left(\mathbb{E}_{1}\right)$. Since $\phi_{\mu} \eta_{\mu}=\beta$ we see easily that $\alpha \eta_{\infty}=\beta$.

It follows from $\alpha \eta_{\infty}=\beta=\alpha \eta$ that $\eta_{\infty}(x)=\eta(x) \cdot c(x)$ and $c(x)$ is in the center, $\eta(C)$. Since for each $x \in X c(x)$ is a central element, $c$ is an algebraic homomorphism of $X$ into $\eta(C)$. Let $R$ be the image of $X$ under $c$. If $R$ is not trivial we could choose an invariant subgroup $H_{\mu}^{1}$ of $\eta(C)$ such that $\eta(C) / H_{\mu}^{1}$ is finite and $H_{\mu}^{1} \cap R \neq R$. Then $R / R \cap H_{\mu}^{1} \neq e$ and we should then have a proper invariant subgroup of $X$ whose factor group is finite. This would contradict Theorem 2.1 and consequently $\eta_{\infty}=\eta$. Since $\eta$ is then continuous we see that the topology $\mathfrak{S}_{1}$ on $G$ is induced by the topology $\mathbb{S}$ on $X$ which is just the topology $\sqrt{5}$ on $G$, and hence the theorem.

4. We consider in this section the case of abelian groups. We prove first a general structure theorem and point out some examples which contrast this case with the case of the last section.

Theorem 4.1. Let $G$ be a compact, connected abelian group. Then

$$
G=H+K \text { (topological direct sum), }
$$

where $H$ is a compact, connected group in which the elements of finite order in $G$ are dense, and $K$ is a topological direct product of a certain number of copies of 
the character group of the rational numbers (the rational numbers considered here in the discrete topology).

Proof. Let $H^{\prime}$ denote the subgroup of elements of finite order in $G$, and $H$ the closure of $H^{\prime}$ in $G$. Since $G$ is connected and compact, the group $G$ is divisible $\left[4\right.$, p. 55] and then clearly $H^{\prime}$ is a divisible group. It is easily seen that the closure of a divisible group in a compact group is divisible since $x \rightarrow n \cdot x$ is a continuous function, and consequently $H$ is divisible. According to $[4$, p. 8] $H$ is then algebraically a direct summand of $G$ and we have

$$
\boldsymbol{G}=\boldsymbol{H}+\boldsymbol{K} \text { (algebraic direct sum), }
$$

and furthermore $K$ is divisible since $G$ is. Then the underlying group of the topological group $G / H$ is isomorphic to $K$. Since $H^{\prime} \subset H, H \cap K=0, K$ has no nontrivial elements of finite order and consequently $K$ is a vector space over the rational numbers $[4$, p. 10$]$. Since the topological group $G / H$ is compact and connected, its character group, $X$, has no elements of finite order. Furthermore, $X$ is divisible. For if $f: G / H \rightarrow C$, where $C$ is the circle in its usual topology, is a continuous homomorphism, then the continuous homomorphism $g: G / H \rightarrow C$ defined by $g(x)=f(x / n)$ shows that $X$ is divisible. Now $X$ can be considered as the subgroup of the character group $G^{\wedge}$ of $G$ which annihilates $H$, and since $X$ is a divisible group, $X$ is a direct summand of $G^{-}$. Then we have

$$
G^{\widehat{C}}=X+P \text {. }
$$

By duality, $H$ is the character group of $G^{-} / X \cong P$. It follows from (4.2) that the character group of $G^{\uparrow}$, which is of course $G$, decomposes into the topological direct sum of two closed subgroups which have for their character groups $X$ and $P$ respectively. Clearly the character group of $X$ contains no elements of finite order since $X$ is divisible. Since the topological direct summand of $G$ corresponding to the character group of $P$ must contain all the elements of finite order it must be $H$. That the character group of $X$ has the prescribed form follows from the representation $X=\sum_{\mu \in N} R_{\mu}$.

REMARKs. With respect to the development of III, we give the following examples to show that in the abelian case nothing like uniqueness prevails, even up to topological isomorphism.

1. Let $\left\{p_{n}\right\}$ be some ordering of the primes. Consider the inverse system $\left\{S_{n}{ }^{\prime}, \alpha_{n}\right\}$ where for each $n, S_{n}^{1}$ is topologically isomorphic to the usual circle group, $S^{1}$, and $\alpha_{n}: S_{n}^{1} \rightarrow S_{n-1}^{1}$ is given by $\alpha_{n}(x)=p_{n} x$. The inverse limit can be seen to be algebraically isomorphic to $S^{1}$ but cannot be topologically isomorphic to $S^{1}$.

2. Let $K$ be the character group of a finite or countable direct sum of copies of the rational numbers in the discrete topology. Then $S^{1}+K$ (topological direct sum) is algebraically isomorphic to $S^{1}$ (see $[2 ; 3 ; 4]$ ). 


\section{REFERENCES}

1. S. Eilenberg and N. Steenrod, Foundations of algebraic topology, Princeton University Press, 1952.

2. D. K. Harrison, On infinite Abelian groups, Ann. of Math. vol. 69 (1959) pp. 366-391.

3. A. Hulanicki, Algebraic characterization of Abelian divisible groups which admit compact topologies, Fund. Math. vol. 44 (1957).

4. I. Kaplansky, Infinite Abelian groups, University of Michigan Press, 1954.

5. D. Montgomery and L. Zippin, Topological transformation groups, New York, Interscience Publishers, 1955.

6. L. Pontrjagin, Topological groups, Princeton University Press, 1946.

7. N. Steenrod, Universal homology groups, Amer. J. Math. vol. 58 (1936) pp. 661-701.

8. B. L. van der Waerden, Stetigkeitsatze für halbeinfache Liesche Gruppen, Math. Z. vol. 36 (1932-1933) pp. 780-786.

UNIVERSITY OF Notre DAME,

Notre Dame, Indiana

InSTITUTE fOR Advanced StUdy,

Princeton, New Jersey 